\title{
Inventário de Arnett de Busca de Sensações (AISS): testando diferentes modelos fatoriais
}

\author{
Valdiney Veloso Gouveia' - Universidade Federal da Paraíba, João Pessoa, Brasil \\ Carlos Eduardo Pimentel - Universidade de Brasilia, Brasilia, Brasil \\ Rildésia S. V. Gouveia - Centro Universitário de João Pessoa, João Pessoa, Brasil \\ Leogildo Alves Freires - Universidade Federal da Paraíba, João Pessoa, Brasil \\ Rebecca Alves Aguiar Athayde - Universidade Federal da Paraíba, João Pessoa, Brasil \\ Rafaella de Carvalho Rodrigues Araújo - Universidade Federal da Paraíba, João Pessoa, Brasil
}

\begin{abstract}
Resumo
O estudo do traço de busca de sensações permite explicar diversos comportamentos de risco. Considerando sua relevância e a ausência de medida específica no Brasil, decidiu-se adaptar o Inventário de Arnett de Busca de Sensações. Este é formado por 20 itens, medindo dois fatores: novidade e intensidade. Participaram da pesquisa 279 estudantes do ensino médio de escolas particulares e públicas, com idade média de 17 anos, igualmente distribuídos quanto ao sexo, provenientes de João Pessoa $(n=108)$ e Teresina $(n=171)$. Por meio de análises fatoriais confirmatórias, testaram-se quatro modelos, segundo o número de fatores (um ou dois) e itens (12 ou 20). Os resultados mostraram que o modelo bifatorial reduzido apresentou melhores índices de ajuste. Os fatores e a pontuação total desse instrumento apresentaram consistência interna aceitável, estando correlacionados com sexo e idade dos participantes. Tais resultados justificam a utilização dessa medida em estudos futuros. Palavras-chave: Busca de sensação, Personalidade, Inventário, Validade, Análise fatorial.
\end{abstract}

\section{Arnett Inventory of Sensation Seeking (AISS): testing different factorial models}

\begin{abstract}
The study of sensation seeking trait is able to explain several risky behaviors. Considering its relevance and the lack of specific instruments to measure it in Brazil, it was decided to adapt the Arnett Inventory of Sensation. It is composed by 20 items, measuring two factors: novelty and intensity. Participants were 279 high school students of private and public schools from João Pessoa $(n=108)$ and Teresina $(n=171)$. They had mean age of 17 years old, being equally distributed for sex. Using confirmatory factor analysis, four models were tested according to the number of factors (one or two) and items (12 or 20). Results showed that the reduced bifactorial model presented the best fit indices. The factors and the total score of this instrument presented acceptable internal consistency, being correlated with sex and age. These results justify the use of this instrument in subsequent studies.

Keywords: Sensation seeking, Personality, Inventory, Validity, Factor analysis.
\end{abstract}

O construto busca de sensações está entre os mais importantes no âmbito da Psicologia da Personalidade, contribuindo para compreender e predizer comportamentos e interações sociais (Arnett, 1994; Zuckerman, 1979, 1994), como se verá no decorrer deste texto. Seu emprego tem sido evidente em diferentes áreas de interesse, como estilos de percepção e cognição, experiências de vida e psicopatologia (Zuckerman, Buchsbaum \& Murphy, 1980).

Zuckerman (1994, p. 1) definiu busca de sensações como "um traço que descreve a tendência para procurar sensações e experiências novas variadas, complexas e intensas, e a disposição para correr riscos com a finalidade de satisfazer tais experiências". Arnett (1994), por sua vez, conceituou a busca de sensações como uma predisposição ou um potencial que depende de outras características de personalidade para sua manifestação, assim como de ambientes de

1 Endereço para correspondência:

Universidade Federal da Paraíba - CCHLA

Departamento de Psicologia - 58 051-900 - João Pessoa - PB

E-mail: vvgouveia@gmail.com socialização. Mais especificamente, esse autor destaca que "a busca de sensações não é apenas um potencial para a tomada de riscos, mas compreende uma qualidade da busca de intensidade e novidade na estimulação sensorial" (Arnett, 1994, p. 290).

Arnett (1994), complementando sua definição, indica que tal traço pode ser refletido em esferas múltiplas da vida, podendo se expressar de formas variadas, tanto por meio de comportamentos socialmente aceitos como antissociais. Nessa mesma linha, Arnett (1998) sugere que as situações que envolvem comportamentos de risco atraem pessoas que têm potencialmente alta busca de novidade e intensidade, particularmente adolescentes. Explica, ainda, que as pessoas mais prováveis de se casarem e terem filhos apresentam níveis baixos de busca de sensação, ao passo que demonstram graus elevados de religiosidade.

Zuckerman e colaboradores exemplificaram os extremos da dimensão busca de sensações com referência a dois cachorros caracterizados por Pavlov. O primeiro (típico de buscador alto de sensações) era expansivo, vivaz, 
extrovertido e mantinha contato com os humanos; e o segundo (típico de buscador baixo de sensações) era cauteloso, introvertido, evitando contato com humanos, novas situações e estimulações.

A partir de trabalhos sobre reações diante de situações de privação de estímulos, Zuckerman (1979) conjeturou a existência de uma variável de personalidade referida precisamente à necessidade de experiências e sensações variadas, novas e complexas e a disposição para assumir riscos físicos e sociais a fim de conseguir tais experiências. Em realidade, essa hipótese tem sido desenvolvida desde a segunda metade dos anos 1960, procurando analisar e aprimorar a teoria deste traço de personalidade (Zuckerman, 1994). Ele se apresenta em todos os indivíduos, variando em intensidade, atingindo picos entre 16 e 20 anos de idade; engloba atributos como desinibição, busca de emoções (entusiasmo), necessidade de aventura, de novas experiências e suscetibilidade ao tédio (Carton, Jouvent \& Widlöcher, 1994). A busca de sensações está ligada a características biológicas; o "buscador de sensações" é retratado por apresentar uma atividade alta dos sistemas cerebrais encarregados de regular a sensibilidade às experiências gratificantes (Ames, Zogg \& Stacy, 2002; Zuckerman, 1993). Quanto às bases biológicas da personalidade, uma revisão de pesquisas (a maioria com delineamento correlacional) com animais e humanos mostrou convergência entre busca de sensações e monoamina oxidase (MAO), reflexo de orientação, níveis de hormônios gonadais e potenciais de evocação (Zuckerman \& cols., 1980). Esses correlatos biológicos mostraram sua importância também para entender comportamentos antissociais (Raine, 2008), a exemplo do uso de drogas e agressão.

Dentre as características comuns aos altos buscadores de sensações estão as atitudes positivas em relação à emoção ou alegria, e expressões mais desinibidas. Assim, espera-se que a busca de sensações prediga a abertura à mudança, as atitudes receptivas em relação às novas experiências, e a habilidade para tolerar sensações e ideias que são comumente estranhas para a maioria das pessoas. Os altos buscadores de sensações são também considerados como mais sociáveis, impetuosos, assertivos, atrevidos e demonstram menos medo (Aluja, 1989, 1990; Zuckerman, 1983, 1994; Zuckerman, Eysenck \& Eysenck, 1978). Além dos estudos de Zuckerman e seus colaboradores, outras pesquisas também indicaram variáveis correlatas ao construto de busca de sensações.

Dentre os vários estudos realizados com busca de sensações e comportamentos antissociais ou de risco (Arnett, 1994; Arnett \& Balle-Jensen, 1993;
Formiga, Aguiar \& Omar, 2008; Greene, Krcmar, Walters, Rubin \& Hale, 2000; Heaven, 1996; Santos, 2008; Vasconcelos, Gouveia, Pimentel \& Pessoa, 2008), foram encontradas correlações positivas com o uso de substâncias psicoativas (Crawford, Pentz, Chou, Li \& Dwyer, 2003), incluindo álcool (Andrew \& Cronin, 1997), tabaco (Greene \& cols., 2000), maconha, haxixe, anfetaminas e LSD (para uma revisão de estudos ver também Zuckerman \& cols., 1980).

Arnett (1992a, 1992b) desenvolveu a teoria da socialização ampla e específica, a qual explica, com base na busca de sensações, que os comportamentos de risco são mais comuns na adolescência. Foi constatado também que os buscadores de sensações demonstravam maior preferência por músicas anticonvencionais (por exemplo, heavy metal; Arnett, 1991; Litle \& Zuckerman, 1986; McNamara \& Ballard, 1999), davam mais importância a valores de experimentação (por exemplo, prazer, emoção; Vasconcelos, 2004), aderiam a práticas sexuais de risco (por exemplo, não usar preservativo, ter múltiplos parceiros; Arnett, 1990; Greene \& cols., 2000) ou indicavam interesse acentuado por jogos de azar (McDaniel \& Zuckerman, 2003).

Por fim, constatou-se que algumas variáveis demográficas se correlacionaram com a busca de sensações. Por exemplo, no que tange ao sexo, já foi verificado que os adolescentes do sexo masculino apresentaram maior tendência para busca de sensações. No caso da idade, constatou-se sua correlação negativa com este construto, indicando que pessoas mais velhas são menos prováveis de serem buscadoras de sensações (Arnett, 1994; Ledesma, Poó \& Peltzer, 2007; Roth \& Herzberg, 2004; Zuckerman, 1994; Zuckerman \& cols., 1980).

De acordo com o anteriormente descrito, é possível encontrar diversas pesquisas com o construto busca de sensações. No âmbito internacional, ao introduzir o termo "sensation seeking" no site de busca da American Psychological Association (2009), APA PsycNETTM, encontraram-se 2.987 resultados referentes a esse termo em qualquer parte das publicações (por exemplo, artigos, capítulos de livros, livros e teses). Nesse caso, ficou evidente sua importância e aplicabilidade, sendo considerado em revistas científicas de diversas áreas temáticas, como personalidade, psicologia social, psicologia da música, avaliação psicológica, psicologia clínica, psiquiatria, adolescência, enfermagem, comunicação, psicofarmacologia, sexualidade, etc.

A situação parece algo diversa e menos favorável no contexto nacional. Por exemplo, empregando o termo correspondente em português 
(busca de sensações) no índice de assuntos dos Periódicos Eletrônicos de Psicologia (2009), surgiram apenas dois registros. Repetindo esta busca no Scientific Eletronic Library Online (2009), restringindo para o Brasil, pelo método de busca integrada, encontraram-se cinco resultados, mas apenas um artigo tratou diretamente do conceito (apresentando informações a respeito no seu resumo). Ampliando-se a busca para o Google Acadêmico (2009), adotando o mesmo termo em qualquer lugar do artigo, verificou-se que diversos autores citaram ou pesquisaram esse construto. Porém, ao se considerar o construto como parte do título do artigo, apareceram dois trabalhos de congressos nacionais em psicologia. Portanto, pode-se concluir que muito há ainda que estudar a respeito no contexto nacional. Contudo, antes de qualquer tentativa nessa direção, parece crucial contar com uma medida adequada sobre esse construto. Isso motivou ter em conta o Inventário de Busca de Sensações de Arnett (AISS; Arnett, 1994), que compreende um instrumento de aplicação rápida, tipo lápis e papel, auto aplicável e que reúne parâmetros psicométricos aceitáveis.

Inventário de Busca de Sensações de Arnett (AISS)

Considerando a escassez evidenciada de pesquisas na literatura da psicologia no Brasil, o presente estudo objetivou adaptar uma escala de busca de sensações, que poderá ser utilizada em pesquisas futuras. Especificamente, descrevem-se neste artigo evidências de validade fatorial e consistência interna do Inventário de Busca de Sensações de Arnett (AISS; Arnett, 1994).

Algumas razões motivaram a escolha desse construto e do inventário em pauta. Primeiramente, existe uma classe ampla de comportamentos que podem ser explicados pelo construto busca de sensações, como avaliado por meio do AISS. Em segundo lugar, destaca-se que esta medida tem a vantagem de ser relativamente breve, com coeficientes aceitáveis de consistência interna (úteis para o contexto da pesquisa). Finalmente, a medida correspondente se correlaciona com outra classicamente empregada na literatura (a Escala de Busca de Sensações; SSS), mas esta conta com o dobro de itens. Destaca-se que a aplicabilidade mais evidente do AISS é a possibilidade de explicar (predizer) comportamentos que expressam a busca por novidade e intensidade na estimulação sensorial (Arnett, 1994).

Como guia para a construção de uma medida de busca de sensações, Arnett (1994) destacou que sua escala deveria medir duas de suas dimensões principais: intensidade e novidade da estimulação dos sentidos. Portanto, verifica-se que essa medida parte de um modelo teórico parcimonioso. Ele considerou que os comportamentos ilegais ou de violação de normas não deveriam fazer parte de sua medida. Este também é um argumento importante para essa medida, pois não tratou de comportamentos que podem ser previstos pelo traço, mas que não fazem parte da sua definição, como o uso de drogas. Outro critério utilizado para a construção dessa escala foi que não deveriam ser formulados itens intrinsecamente relacionados com a idade, e que a escala de resposta deveria ser de tipo Likert, considerada mais comumente empregada e de fácil compreensão. Em suma, Arnett (1994) utilizou esses critérios para construir seu inventário com base nas críticas feitas à medida de Zuckerman (1993).

Por meio de duas pesquisas com estudantes adolescentes e adultos da população geral em Atlanta (Estados Unidos), o autor encontrou correlações da sua medida com a Escala de Busca de Sensações (SSS), Forma V, de Zuckerman, variando de 0,08 a 0,47 com as subescalas e de 0,15 a 0,46 com a escala total (Arnett, 1994). Foram encontradas correlações positivas com vários comportamentos de risco, como uso de maconha, fazer sexo com algum desconhecido, vandalismo, roubo e dirigir de modo arriscado (drogado ou em alta velocidade). Arnett destacou que tais correlações foram mais fortes com o AISS do que com a SSS. Foram encontrados coeficientes de consistência interna aceitáveis para o AISS, como seguem: 0,70 (escala total), 0,64 (fator intensidade) e 0,51 (fator novidade). Seus fatores específicos, isto é, intensidade e novidade, mostraram-se positivamente correlacionados entre si $(r=0,41, p<0,001)$.

Esse primeiro estudo também demonstrou que os adolescentes do sexo masculino apresentaram pontuações mais altas do que os do sexo feminino, considerando tanto a pontuação total da escala como a de seu fator específico denominado intensidade. O segundo estudo, relatado na mesma publicação (Arnett, 1994), corroborou as correlações do primeiro, acrescentando a de busca de sensações com agressividade e assinalando diferenças nas pontuações de adolescentes e adultos.

O AISS também foi recentemente testado em amostra de 1.236 estudantes alemães do ensino médio (Roth \& Herzberg, 2004), verificando-se, por meio de análises fatoriais confirmatórias, que o modelo bifatorial proposto por Arnett (Modelo 1) apresentou itens com pesos fatoriais não-significativos e índices de ajuste considerados por tais autores como não satisfatórios $\quad\left(\chi^{2} /\right.$ g.l. $=4,03, \quad p<0,001, \quad G F I=0,88$, $A G F I=0,86$ e RMSE $A=0,07)$. Considerando os itens com pesos estatisticamente significativos, Roth e Herzberg testaram um modelo bifatorial reduzido, que eles julgaram apresentar índices de ajuste mais 
satisfatórios $\quad\left(\chi^{2} /\right.$ g.l. $=4,62, \quad p<0,001, \quad G F I=0,94$, $A G F I=0,90$ e $R M S E A=0,08)$.

Portanto, para chegar a esses resultados Roth e Herzberg (2004) excluíram os itens 3, 5, 13, 15 e 17, do fator novidade, e os itens 2,10, e 14, do fator intensidade, propondo um modelo reduzido. Esse modelo foi testado em uma amostra independente, confirmandose os índices encontrados previamente $\left(\chi^{2} /\right.$ g.l. $=4,18$, $p<0,001, G F I=0,94, A G F I=0,91$ e RMSE $A=0,07)$. Essa versão reduzida, composta por 12 itens, apresentou os seguintes coeficientes de consistência interna (alfas de Cronbach, $\alpha$ ): 0,60 (escala total), 0,57 (novidade) e 0,59 (intensidade), que são considerados por tais autores como suficientes para o contexto da pesquisa. Esses índices são consistentes com os anteriormente apresentados por Roth (2003) com uma amostra de 205 trabalhadores alemães. Esse autor também verificou correlações entre o AISS e uma medida alemã de desejabilidade social, semelhante à Escala de Desejabilidade de Marlowe-Crowne (Crowne \& Marlowe, 1960; Gouveia, Guerra, Souza, Santos \& Costa, 2009). Roth, Schumacher e Brähler (2004) também utilizaram o AISS, verificando que essa medida conseguiu diferenciar homens e mulheres no que diz respeito às suas pontuações em busca de sensações. Ressalta-se que seus índices de consistência interna foram favoráveis para os propósitos de pesquisa: 0,64 (novidade), 0,73 (intensidade) e 0,80 (escala total).

Considerando o anteriormente detalhado, justifica-se o empreendimento de conhecer os parâmetros psicométricos do AISS no contexto brasileiro. Especificamente, busca-se verificar sua validade de construto (estrutura fatorial e consistência interna) por meio de análise fatorial confirmatória (DiStefano \& Hess, 2005; Waktins, 1989) e alfa de Cronbach, como recomendado na literatura (Anastasi \& Urbina, 2002; Nunnally, 1991; Pasquali, 2003).

\section{Método}

\section{Participantes}

Contou-se com a participação de 279 estudantes do ensino médio, com idades entre 13 e 38 anos $(m=17,3, d p=3,47 ; 89 \%$ com até 20 anos de idade), sendo a maioria de escolas públicas $(65,7 \%)$ e solteira $(65,6 \%)$, igualmente distribuídos quanto ao sexo. Esses participantes foram de duas cidades do Nordeste brasileiro: João Pessoa (PB; $n=108)$ e Teresina (PI, $n=171)$. Tratou-se de uma amostra de conveniência, não-probabilística, tendo participado aqueles que, presentes em sala de aula, concordaram voluntariamente em fazer parte do estudo.

\section{Instrumento}

Os participantes responderam perguntas demográficas (cidade, escola, idade e sexo) e a medida de busca de sensações, descrita a seguir:

Inventário de Busca de Sensações de Arnett (AISS; Arnett, 1994). Essa é uma medida de autorrelato, auto-administrável, tipo lápis e papel, composta por 20 itens distribuídos igualmente em dois fatores: novidade (itens 1, 3, 5, 7, 9, 11, 13, 15, 17 e 19; por exemplo, item 07. Eu acho divertido e excitante atuar ou falar na presença de um grupo) e intensidade (itens $2,4,6,8,10,12,14,16,18$ e 20; por exemplo, item 20. Deve ser excitante estar em uma batalha durante a guerra). Os itens 2, 3, 6, 10, 13 e 17 são invertidos, estratégia utilizada pelo autor para evitar viés de resposta (Arnett, 1994). Cada item é respondido em escala de tipo Likert, na qual a pessoa deve afirmar em que medida ele a descreve, considerando quatro opções de respostas, variando de $\mathbf{1}=$ Descreve-me muito bem a $\mathbf{4}=$ Não me descreve totalmente.

A versão original em inglês do AISS foi traduzida para o português com a colaboração de um painel de três pesquisadores bilíngues, procurando alcançar o consenso em cada item. Essa versão em português foi posteriormente administrada a uma amostra de dez estudantes do ensino médio, procurando conhecer se a redação dos itens, o formato da escala de resposta e as instruções de como proceder eram compreensíveis. Em razão das observações efetuadas, decidiu-se inverter a escala de resposta, que passou a ser $\mathbf{1}=$ Não me descreve totalmente e 4= Descreveme muito bem, e realizar pequenas modificações linguísticas, tornando a redação mais facilmente compreensível para os participantes. Portanto, contouse com a versão experimental dessa medida, significando a maior pontuação evidência de maior busca de sensações. O leitor interessado poderá obter uma cópia escrevendo a um dos autores.

\section{Procedimento}

Com o fim de administrar a versão em português do AISS, contataram-se diretores de escolas públicas e privadas do ensino médio. Inicialmente, buscou-se levantar o nome das escolas na cidade (mesmo procedimento para João Pessoa e Teresina). Depois de consultados seus diretores, agendava-se a aplicação dos questionários. A coleta de dados ocorreu em contexto coletivo de sala de aula, porém as respostas foram dadas individualmente pelos participantes do estudo. Os questionários foram administrados por dois bolsistas do PIBIC de Psicologia de uma instituição pública da Paraíba e por uma psicóloga colaboradora da pesquisa no Piauí. 
Os aplicadores se identificavam como membros de um grupo de pesquisa interessado em conhecer como os jovens pensam e agem no seu dia a dia. Com essa prerrogativa, os aspectos éticos para condução de pesquisas com seres humanos foram considerados, tendo sido os participantes informados que sua participação era voluntária, que ninguém seria nominalmente identificado, que poderiam declinar da participação a qualquer momento da pesquisa e que o feedback seria realizado em forma de relatório técnico apresentado à escola. Cada diretor assinou um termo de consentimento livre e esclarecido, autorizando a participação dos estudantes de sua escola. Cerca de 20 minutos foram suficientes para concluir a participação no estudo.

\section{Análise dos dados}

A digitação e análise dos dados foram realizadas com o SPSS (versão 15). Foram conduzidas análises descritivas (média, desvio padrão, percentuais) e análises inferenciais. Esse programa permitiu obter correlações de Pearson, realizar análise de variância (ANOVA), análise multivariada de variância (MANOVA), calcular os alfas de Cronbach e a homogeneidade (correlação média inter itens) para os fatores do AISS. Com o fim de conhecer evidências de sua validade fatorial, foram realizadas análises fatoriais confirmatórias (CFA) com o AMOS (versão 7). Nesse caso, consideraram-se os seguintes indicadores de ajuste dos modelos (Byrne, 2001; Garson, 2003):

- Razão $\chi^{2} /$ g.l. Este índice, isto é, o qui-quadrado dividido pelos graus de liberdade, deve ser preferencialmente entre 2 e 3 (ou menos), admitindose valores de até 5 .

- GFI e AGFI. São índices absolutos de ajuste, significando Goodness-of-Fit Index (GFI) e Adjusted Goodness-of-Fit Index (AGFI), devendo apresentar valores próximos de 1 , preferencialmente superiores a 0,90 .

- RMSEA. O valor da Root-Mean-Square Error of Approximation é tido como melhor quanto mais próximo de zero, preferencialmente não maior do que 0,05, mas tolerando-se até 0,10. O Pclose testa a hipótese de RMSEA $>0,05$, $\operatorname{com} p>0,05$ sugerindo sua rejeição, sendo modelo, portanto, considerado proximamente ajustado.

- ECVI, CAIC e Teste de Diferença entre QuiQuadrados $\left(\Delta \chi^{2}\right)$. O Expected Cross-Validation Index (ECVI) e Consistent Akaike Information Criterion (CAIC) são úteis para a comparação de modelos, considerando-se como mais adequado aquele que apresenta menores valores de ECVI e CAIC. No caso do $\Delta \chi^{2}$, quando significativo, indica melhor ajuste do modelo com menor valor de $\chi^{2}$.

\section{Resultados}

Com propósitos estritamente didáticos, os resultados são descritos em três blocos principais. Inicialmente, comprovam-se os modelos fatoriais da medida de busca de sensações. Posteriormente, definida a estrutura resultante, são avaliadas a homogeneidade dos itens (isto é, correlação média inter itens, $\mathrm{r}_{\mathrm{i} . \mathrm{i}}$ por fator) e a consistência interna (alfa de Cronbach) dos fatores. Finalmente, os fatores do AISS são comparados/correlacionados com as variáveis cidade, sexo, escola e idade dos participantes.

\section{Análises fatoriais confirmatórias}

De acordo com a literatura, são possíveis ao menos quatro modelos fatoriais para o Inventário de Arnett de Busca de Sensações, a saber: (1) modelo original $\left(M_{1}\right)$, reunindo dois fatores (novidade $\mathrm{e}$ intensidade), cada um com dez itens; (2) modelo original unifatorial $\left(M_{2}\right)$, com os 20 itens saturando em um mesmo fator geral de busca de sensações; (3) modelo reduzido bifatorial $\left(M_{3}\right)$, composto por 12 itens, cinco em um fator (novidade) e sete em outro (intensidade); e (4) modelo reduzido unifatorial $\left(M_{4}\right)$, em que os 12 itens saturam em um fator geral de busca de sensações. Esses modelos foram testados com o AMOS 7, considerando-se como entrada a matriz de covariância inter itens e adotando o estimador ML (Máxima Verossimilhança). Os resultados são mostrados na Tabela 1.

Tabela 1 - Índices de ajuste dos modelos bifatorial e unifatorial (originais e reduzidos) do AISS

\begin{tabular}{lccccccc}
\hline Modelo & $\chi^{2 / g .1 .}$ & GFI & AGFI & RMSEA (IC90\%) & Pclose & ECVI & CAIC \\
\hline Bifatorial (20 itens) & 1,83 & 0,90 & 0,87 & $0,055(0,045-0,064)$ & 0,208 & 1,41 & 581,14 \\
Unifatorial (20 itens) & 1,87 & 0,90 & 0,87 & $0,056(0,046-0,065)$ & 0,144 & 1,43 & 583,73 \\
Bifatorial (12 itens) & 1,82 & 0,95 & 0,92 & $0,054(0,037-0,071)$ & 0,326 & 0,53 & 262,08 \\
Unifatorial (12 itens) & 2,03 & 0,94 & 0,91 & $0,061(0,044-0,077)$ & 0,137 & 0,57 & 268,61 \\
\hline
\end{tabular}

De acordo com essa tabela, os melhores índices de ajuste são observados para o modelo 3 (bifatorial reduzido): $\quad \chi^{2} /$ g.l. $=1,82, \quad$ GFI $=0,95$,
$A G F I=0,92, R M S E A=0,054(I C 90 \%=0,037-0,071) \mathrm{e}$ Pclose $=0,326$. Reforçando a natureza bidimensional do construto avaliado, os índices de ajuste para a estrutura 
com dois fatores com o conjunto original de itens (modelo 1) podem ser encarados como meritórios: $\chi^{2} /$ g.l. $=1,83, G F I=0,90, A G F I=0,87$, RMSE $A=0,055$ $(I C 90 \%=0,045-0,064)$ e Pclose $=0,208$. O modelo 3 foi o que obteve, comparativamente aos outros três testados, os menores valores de ECVI $(0,53)$ e CAIC (262,08); confirmando esse achado, ele apresentou menor qui-quadrado do que o modelo $4\left[\Delta \chi^{2}(1)=13,16\right.$, $p<0,001]$. O modelo 3 é representado na Figura 1.

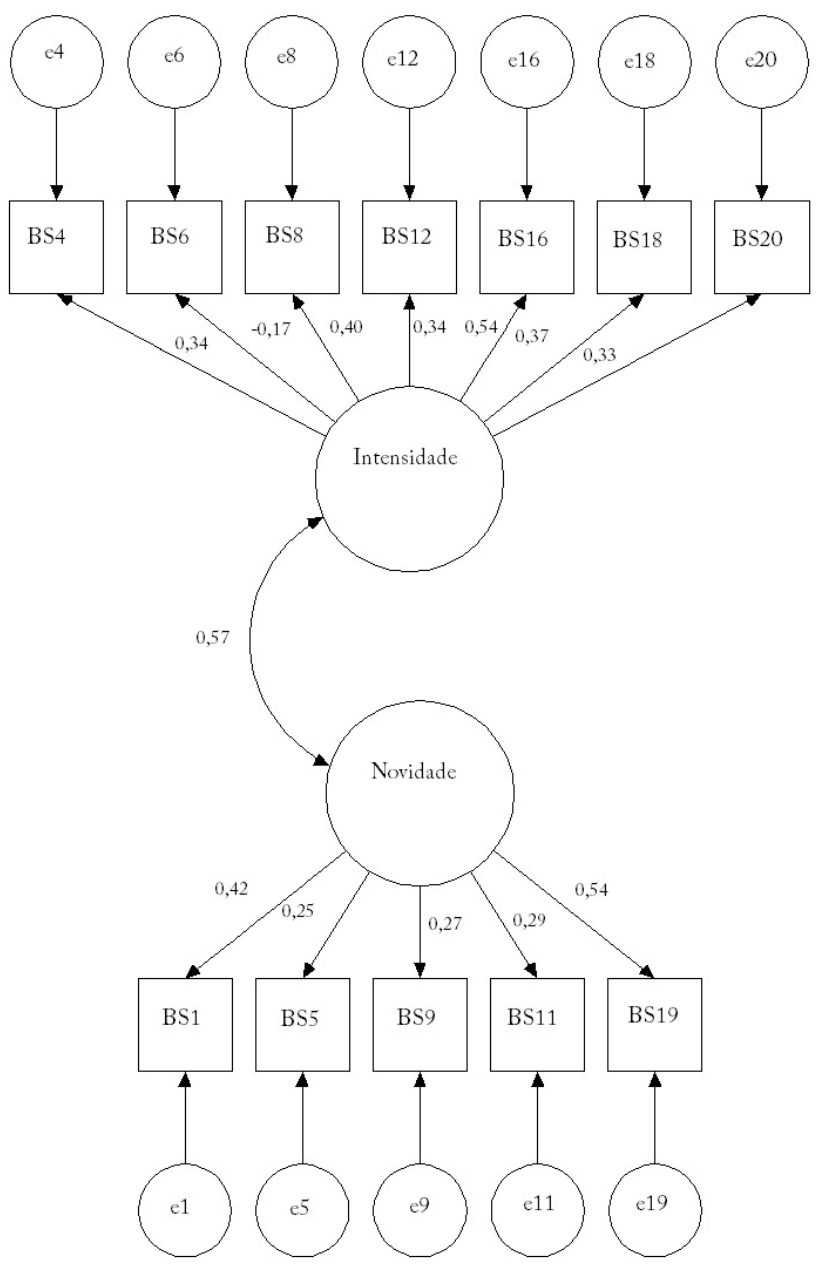

Figura 1 - Estrutura fatorial do Inventário de Arnett de Busca de Sensações

Destaca-se que todas as saturações (lambdas) dos itens da Figura 1 foram estatisticamente diferentes de zero $(\lambda \neq 0 ; z>1,96, p<0,05)$, variando de $-0,17$ (Item 6. Eu não assisto filmes que são assustadores on cheios de suspense) a 0,54 (item 16. Acho que seria interessante ver um acidente de carro acontecendo; e item 19. Se fosse possivel visitar outro planeta ou ir à lua gratuitamente, gostaria de estar entre os primeiros na fila para se inscrever). Os dois fatores resultantes se mostraram correlacionados (covariando) entre si $(\phi=0,57)$, indicando que, embora sejam legítimos tais fatores, o construto busca de sensações se revela integrado.

\section{Homogeneidade e Consistência Interna}

Tomando em conta o modelo 3, que se revelou mais adequado, checou-se a homogeneidade (correlação média inter itens) e a consistência interna (alfa de Cronbach) de seus dois fatores. O fator intensidade apresentou homogeneidade de 0,15, variando de 0,10 (item 6. Eu não assisto filmes que são assustadores ou cheios de suspense) a 0,26 (item 16. Acho que seria interessante ver um acidente de carro acontecendo). Compondo-se de sete itens, sua consistência interna foi 0,51 . No caso do fator novidade, composto por cinco itens, sua homogeneidade foi 0,22, variando de 0,20 (item 5. Considero melhor planejar uma viagem o menos possivel e apenas aproveitá-la como ela ê) a 0,24 (item 19. Se fosse possivel visitar outro planeta ou ir à lua gratuitamente, gostaria de estar entre os primeiros na fila para se inscrever), com consistência interna de 0,42 . Quando são tidos em conta os 12 itens que compõem tais fatores, observa-se alfa de Cronbach de 0,56. 
Fatores de busca de sensaçoes e seus correlatos

Uma vez que os fatores novidade e intensidade se correlacionaram diretamente entre si $(r=0,24$, $p<0,001)$, além das pontuações específicas destes fatores, calculou-se a pontuação total para a escala, reunindo os 12 itens da versão abreviada do AISS. Nesse sentido, como primeiro passo, efetuou-se uma ANOVA em que o sexo, a escola e a cidade (lugar de residência) dos participantes entraram como variáveis antecedentes, sendo considerada como variável critério a pontuação total da medida de busca de sensações. Não se encontrou qualquer diferença estatisticamente significativa com respeito às três variáveis antecedentes consideradas: sexo $[F(25)=1,30, \quad p=0,16]$, escola $[F(25)=0,75, p=0,81]$ e cidade $[F(25)=1,04, p=0,41]$.

Posteriormente, consideraram-se as variáveis antecedentes anteriormente listadas, assumindo-se as pontuações para os fatores intensidade e novidade como variáveis-critério, realizando uma MANOVA. Neste caso, verificou-se efeito principal apenas do sexo no fator intensidade [Lambda de Wilks $=0,90, \quad F(2$, $259)=13,59, p<0,001]$. Especificamente, os homens apresentaram média maior $(m=2,5, d p=0,05)$ do que as mulheres $(m=2,1, d p=0,05)$.

Finalmente, quanto à idade, decidiu-se correlacioná-la com a pontuação total do AISS e seus fatores específicos. Concretamente, constatou-se que esta se correlacionou inversamente com a pontuação total de busca de sensações $(r=-0,17, p<0,01)$ e, sobretudo, com o fator intensidade $(r=-0,22, p<0,001)$; embora a direção da sua correlação com o fator novidade tenha sido na direção esperada $(r=-0,04)$, ela não foi estatisticamente significativa $(p>0,05)$. Portanto, pessoas mais velhas são menos prováveis de obterem pontuações altas em busca de sensações, principalmente no seu fator intensidade.

\section{Discussão}

O objetivo principal deste estudo foi conhecer evidências de validade fatorial e consistência interna do AISS, comparando diferentes versões (12 e 20 itens) e estruturas fatoriais (uni e bifatorial). Confia-se que este tenha sido alcançado. Desse modo, oferece-se como mais adequada uma medida reduzida de busca de sensações, formada por 12 itens e representada por dois fatores: intensidade e novidade. Destaca-se, entretanto, que, embora adequada para os propósitos das análises estatísticas realizadas (DiStefano \& Hess, 2005; Waktins, 1989), a amostra do estudo não é representativa da população em que se inseriu o estudo, sendo descartada a generalização dos resultados quanto aos correlatos de busca de sensações. Cabe ressaltar, não obstante, que esse não foi o propósito principal da pesquisa, que teve natureza psicométrica, enfocando os parâmetros de uma medida específica. Feitas essas considerações, discutem-se os resultados principais.

\section{Estrutura fatorial da medida de Arnett}

O Modelo 1 avaliado retratou a proposta original de Arnett (1994), indicando que o construto busca de sensaçôes poderia ser representado por dois fatores (novidade e intensidade), representados por 20 itens igualmente distribuídos. No geral, os indicadores de ajuste correspondentes não podem ser considerados excelentes, mas devem ser vistos como meritórios, aproximando-se e, inclusive, superando pontos de corte comumente aceitos na literatura, como 0 RMSEA de até 0,08 (Byrne, 2001; Garson, 2003). Contudo, quando inspecionadas as saturações dos itens nos fatores teóricos de pertença, um deles, correspondendo ao fator intensidade, não se mostrou diferente de zero (item 6. Eu não assisto filmes que são assustadores on cheios de suspense). Destaca-se que esse item foi redigido de forma negativa, e este pode ser um aspecto que explique sua baixa saturação (Pasquali, 2003; Ye, 2009).

Parecem evidentes, portanto, os achados que dão apoio empírico para a estrutura bifatorial da medida de busca de sensações ora considerada, isto é, o AISS (Arnett, 1994). Porém, melhores indicadores de ajuste para essa estrutura fatorial foram observados para sua versão reduzida, tendo sido esses resultados coerentes com aqueles relatados por Roth e Herzberg (2004), que levaram a cabo sua pesquisa em outro contexto cultural. Por fim, como esperado, os fatores desse instrumento se mostraram diretamente correlacionados entre si (Arnett, 1994; Roth \& Herzberg, 2004).

\section{Indicadores de confiabilidade dos fatores}

Considerando a estrutura bifatorial reduzida, foi realizada a análise de consistência interna dos fatores específicos e da escala em si, empregando-se o alfa de Cronbach. Essa versão, composta por 12 itens, apresentou índice de precisão aceitável para a escala como um todo, sendo exatamente idêntico aos encontrados por Roth (2003; Roth \& Herzberg, 2004), que teve em conta o mesmo número de itens. No entanto, quando considerada a precisão dos fatores específicos, isto é, novidade e intensidade, não foram encontrados resultados satisfatórios que cumprissem o ponto de corte comumente sugerido (0,70; Nunnally, 1991; Pasquali, 2003). Entretanto, tais resultados também eram esperados, de acordo com alguns estudos prévios (Andrew \& Cronin, 1997; Roth, 2003; Roth \& Herzberg, 2004). 
Considerando o anteriormente dito, mesmo tendo encontrado apoio empírico para a estrutura bifatorial, talvez seja mais indicado utilizar a pontuação total da escala, considerando-a como um todo, com o fim de aferir a magnitude com que as pessoas apresentam o traço de busca de sensações. Esta estratégia de unir os dois fatores do AISS encontra respaldo em Greene e cols. (2000), os quais destacaram que os quatro fatores da SSS também poderiam ser reduzidos a uma só dimensão. Ressalte-se que o estudo de Arnett (1994) também não encontrou índices de consistência interna elevados, sobretudo para seus fatores específicos, embora aceitável para a escala total. Roth e cols. (2004) verificaram que a pontuação total apresenta alfa mais elevado do que os fatores específicos, o que seria esperado em razão do maior número de itens.

Apesar de os alfas de Cronbach não terem sido o que se desejaria, vale salientar que uma característica concreta pode tê-los afetado negativamente: o número de itens de cada fator. Por exemplo, se fosse aumentado para dez itens o número daqueles que compõem cada fator, quantidade que é bastante razoável (Pasquali, 2003), de acordo com a fórmula apresentada por Nunnally (1991, p. 267), poder-se-iam encontrar alfas de Cronbach de 0,59 (novidade) e 0,60 (intensidade).

A homogeneidade dos fatores do AISS pode ser considerada como meritória. Embora não forte, aproxima-se de valores que têm sido recomendados, isto é, próximos de 0,20 (Clark \& Watson, 1995). Além disso, levando-se em conta o tamanho da amostra $(N=268)$, sabe-se que os valores observados (coeficientes de correlação média inter item) diferem estatisticamente de zero $(p<0,05)$, não se devendo, portanto, ao acaso.

\section{Correlatos de busca de sensações}

Foram verificadas diferenças nas pontuações totais do AISS em razão do sexo do respondente, indicando que os homens apresentaram valores mais altos do que as mulheres. Esse achado está em consonância com a literatura internacional sobre esse construto (Zuckerman, 1994; Zuckerman \& cols., 1980). Além disso, corrobora resultados quando da elaboração original dessa medida (Arnett, 1994), assim como estudos relacionados que foram levados a cabo no contexto nacional (Formiga \& cols., 2008) e internacional (Roth \& Herzberg, 2004).

Por fim, coerente também com a literatura, observou-se que quanto maior a idade, menor a pontuação em busca de sensações. Isso endossa a teoria geral de busca de sensações e os estudos prévios que consideraram esta variável demográfica em outros países (Zuckerman, 1994; Zuckerman \& cols., 1980) e com o AISS (Roth \& Herzberg, 2004). Este mesmo padrão de correlação foi recentemente descrito por Ledesma e cols. (2007).

\section{Considerações finais}

Foram testados neste estudo modelos fatoriais para o AISS, seguindo abordagem confirmatória. As evidências encontradas corroboram estudos prévios em relação à validade fatorial e consistência interna dessa medida. Mesmo não tendo sido observados índices elevados de precisão que justifiquem seu emprego no contexto clínico, diagnóstico, esta medida pode ser empregada satisfatoriamente no âmbito da pesquisa. Porém, não se está nesta oportunidade dando a última palavra acerca dos parâmetros psicométricos dessa medida; muito há ainda que ser feito. Portanto, recomenda-se que este inventário reúna provas adicionais de sua adequação psicométrica (Anastasi \& Urbina, 2002; Pasquali, 2003). Por exemplo, conhecer em que medida apresenta validade convergente com o instrumento proposto por Zuckerman (1994), ou se ela apresenta validade discriminante quanto à desejabilidade social, considerada um traço que pode distorcer respostas em medidas que impliquem romper normas sociais (Gouveia \& cols., 2009), como pode ser o caso de buscadores de sensações (Roth, 2003).

Em razão da natureza do construto busca de sensações, um traço de personalidade que, como tal, deveria ser relativamente estável (Aluja, 1989; Zuckerman, 1994), demanda-se checar a precisão do AISS em termos de estabilidade temporal, isto é, testereteste (Pasquali, 2003), considerando diferentes intervalos de tempo (por exemplo, um, três e seis meses). Nesse caso, seria importante estar atento para intervalos mais longos, sobretudo em determinadas faixas etárias, uma vez que a busca de sensações é mais característica de certas fases do desenvolvimento (Zuckerman, 1994). Além disso, tal construto tem origem presumivelmente biológica (Zuckerman \& cols., 1980, Zuckerman 1983, Zuckerman 1993), podendo ser interessante conhecer em que medida as pontuações no AISS se correlacionariam com mensurações biológicas e comportamentos de risco (Arnett, 1994), considerando diferentes contextos, grupos etários e níveis de religiosidade (Arnett, 1998).

Finalmente, tendo em vista a ampla utilização de escalas online na literatura internacional da psicologia (Birnbaum, 2004), seria importante proceder a coletas de dados com esse instrumento via internet. Nesse caso, poder-se-iam montar versões em diversos idiomas, permitindo comparar a estrutura fatorial do AISS em 
pessoas de culturas diferentes, permitindo testar a invariância fatorial dessa medida.

\section{Referências}

Aluja, A. (1989). El rasgo de personalidad "búsqueda de sensaciones": una teoría psicobiológica. Cuadernos de Medicina Psicosomática y Sexología, 11/12, 59-81.

Aluja, A. (1990). La teoría de la Búsqueda de Sensaciones y la teoría de la Personalidad de Eysenck: puntos de contacto. Cuadernos de Medicina Psicosomática y Sexología, 16, 43-50.

American Psychological Association (2009). APA PsycNETTM. Sensation seeking. Obtido em 17 de novembro de 2009 do World Wide Web: $<$ http://psycnet.apa.org.w10002.dotlib.com.br/in dex.cfm? $\mathrm{fa}=$ search.searchResults\&id=048D28E3E39A-5CE9-4E5F-

4C48D77B25A1\&dbTab $=$ all\&page $=5>$.

Ames, S. L., Zogg, J. B. \& Stacy, A. W. (2002). Implicit cognition, sensation seeking, marijuana use and driving behavior among drug offenders. Personality and Individual Differences, 33(7), 1055-1072.

Anastasi, A. \& Urbina, S. (2002). Testagem psicológica. Porto Alegre: ArtMed.

Andrew, M. \& Cronin, C. (1997). Two measures of sensation seeking as predictors of alcohol use among high school males. Personality and Individual Differences, 22(3), 393-401.

Arnett, J. (1990). Contraceptive use, sensation seeking, and adolescent egocentrism. Journal of Youth and Adolescence, 19(2), 171-180.

Arnett, J. (1991). Heavy metal music and reckless behavior among adolescents. Journal of Youth and Adolescence, 20(6), 573-592.

Arnett, J. (1992a). Reckless behavior in adolescents: a developmental perspective. Developmental Review, 12(4), 339-372.

Arnett, J. (1992b). Socialization and adolescent reckless behavior: a reply to Jessor. Developmental Review, 12(4), 391-409.

Arnett, J. (1994). Sensation seeking: a new conceptualization and a new scale. Personality and Individual Differences, 16(2), 289-296.

Arnett, J. (1998). Risk behaviors and family role transitions during the twenties. Journal of Youth and Adolescence, 27(3), 301-320.
Arnett, J. J. \& Balle-Jensen, L. (1993). Cultural bases of risk behavior: Danish adolescents. Child Development, 64(6), 1842-1855.

Birnbaum, M. H. (2004). Human research and data collection via the Internet. Annual Review of Psychology, 55(1), 803-832.

Byrne, B. M. (2001). Structural equation modeling with AMOS: basic concepts, applications, and programming. Mahwah: Lawrence Erlbaum Associates.

Carton S, Jouvent R. \& Widlöcher D. (1994). Sensation seeking, nicotine dependence, and smoking motivation in female and male smokers. Addictive Behavior, 19(3), 219-227.

Clark, L. A. \& Watson, D. (1995). Constructing validity: basic issues in objective scale development. Psychological Assessment, 7(3), 309319 .

Crawford, A. M., Pentz, M. A., Chou, C-P., Li, C. \& Dwyer, J. H. (2003). Parallel developmental trajectories of sensation seeking and regular substance use in adolescents. Psychology of Addictive Behaviors, 17(3), 179-192.

Crowne, D. P. \& Marlowe, D. (1960). A new scale of social desirability independent of psychopathology. Journal of Consulting Psychology, 24(4), 349-354.

DiStefano, C. \& Hess, B. (2005). Using confirmatory factor analysis for construct validation. Journal of Psychoeducational Assessment, 23(3), 225-241.

Formiga, N. S., Aguiar, M. \& Omar, A. (2008). Busca de sensação e condutas antissociais e delitivas em jovens. Psicologia: Ciência \& Profissão, 28(4), 668681.

Garson, G. D. (2003). PA 765 Statnotes: an online textbook. Obtido em 17 de maio de 2005 do World Wide Web: $<$ http://www2.chass.ncsu.edu/garson/pa765/sta tnote.htm>.

Google Acadêmico (2009). Busca de sensações. Obtido em 17 de novembro de 2009 do World Wide Web:

$<$ http://scholar.google.com.br/scholar?as_q=bus $\mathrm{ca}+\mathrm{de}+$ sensações\&num $=10 \& \mathrm{btnG}=$ Pesquisar $+\mathrm{n}$

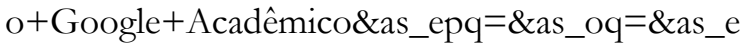
$\mathrm{q}=\&$ as_occt $=$ any\&as_sauthors $=\&$ as_publication $=\&$ as_ylo $=\&$ as_yhi $=\& h l=p t-B R>$.

Gouveia, V. V., Guerra, V. M., Sousa, D. M. F., Santos, W. S. \& Costa, J. M. (2009). Escala de Desejabilidade Social de Marlowe-Crowne: 
evidências de sua validade fatorial e consistência interna. Avaliação Psicológica, 8(1), 87-98.

Greene, K., Krcmar, M., Walters, L. H., Rubin, D. L. \& Hale, L. (2000). Targeting adolescent risktaking behaviors: the contributions of egocentrism and sensation-seeking. Journal of Adolescence, 23(4), 439-461.

Heaven, P. C. L. (1996). Personality and self-reported delinquency: analysis of the "big five" personality dimensions. Personality and Individual Differences, 20(1), 47-54.

Ledesma, R., Poó, F. \& Peltzer, R. (2007). Búsqueda impulsiva de sensaciones y comportamiento de riesgo en la conducción. Avaliação Psicológica, 6(2), 117-125.

Litle, P. \& Zuckerman, M. (1986). Sensation seeking and music preferences. Personality and Individual Differences, 7(4), 575-578.

McDaniel, S. R. \& Zuckerman, M. (2003). The relationship of impulsive sensation seeking and gender to interest and participation in gambling activities. Personality and Individual Differences, 35(6),1385-1400.

McNamara, L. \& Ballard, M. E. (1999). Resting arousal, sensation seeking, and music preference. Genetic, Social, and General Psychology Monographs, 125(3), 229-250.

Nunnally, J. C. (1991). Teoría psicométrica. México: Trillas.

Pasquali, L. (2003). Psicometria: teoria dos testes na psicologia e na educaşão. Petrópolis: Vozes.

Periódicos Eletrônicos de Psicologia (2009). Busca de sensaçoes. Obtido em 17 de novembro de 2009 do World Wide Web: <http://pepsic.bvspsi.org.br/cgi-bin/wxis.exe/iah/>.

Raine, A. (2008). O crime biológico: implicações para a sociedade e para o sistema de justiça criminal. Revista de Psiquiatria do Rio Grande do Sul, 30(1), 5-8.

Roth, M. (2003). Validation of the Arnett Inventory of Sensation Seeking (AISS): efficiency to predict the willingness towards occupational chance, and affection by social desirability. Personality and Individual Differences, 35(6),1307-1314.

Roth, M. \& Herzberg, P. Y. (2004). A validation and psychometric examination of the Arnett Inventory of Sensation Seeking (AISS) in German adolescents. European Journal of Psychological Assessment, 20(3), 205-214.
Roth, M., Schumacher, J. \& Brähler, E. (2005). Sensation seeking in the community: Sex, age and sociodemographic comparisons on a representative German population sample. Personality and Individual Differences, 39(7), 12611271.

Santos, W. S. (2008). Explicando comportamentos socialmente desviantes: uma análise do compromisso convencional e afiliação social. Tese de doutorado. Departamento de Psicologia, Universidade Federal da Paraíba, João Pessoa.

Scientific Eletronic Library Online (2009). Busca de sensaçôes. Obtido em 17 de novembro de 2009 do World Wide Web: $<$ http://search.scielo.org/?q=busca $\% 20$ de $\% 20$ se nsa $\%$ E $7 \%$ F5es\&where $=$ SCL $>$.

Vasconcelos, T. C. (2004). Personalidade, valores e condutas anti-sociais de jovens. Dissertação de mestrado nãopublicada, Departamento de Psicologia, Universidade Federal da Paraíba, João Pessoa.

Vasconcelos, T. C., Gouveia, V. V., Pimentel, C. E. \& Pessoa, V. S. (2008). Condutas desviantes e traços de personalidade: testagem de um modelo causal. Estudos de Psicologia (Campinas), 25(1), 55-65.

Watkins, D. (1989). The role of confirmatory factor analysis in cross-cultural research. International Journal of Psychology, 24(6), 685-701.

Ye, S. Q. (2009). Factor structure of the General Health Questionnaire (GHQ-12): the role of wording effects. Personality and Individual Differences, 46(2), 197-201.

Zuckerman, M. (1979). Sensation seeking: beyond the optimal level of arousal. Hillsdale: Lawrence Erlbaum Associates.

Zuckerman, M. (1983). A biological theory of sensation seeking. Em M. Zuckerman (Ed.). Biological bases of sensation seeking, impulsivity and anxiety (pp. 229-248). Hillsdale: Lawrence Erlbaum Associates.

Zuckerman, M. (1993). Sensation seeking and impulsivity: a marriage of traits made in biology? Em W. G. McCown, J. L. Johnson \& M. B. Shure (Orgs.). The impulsive client: theory, research, and treatment (pp. 71-91). Washington: American Psychological Association.

Zuckerman, M. (1994). Behavioral expressions and biosocial bases of personality. Nova Iorque: Cambridge University Press. 
Zuckerman, M., Eysenck, S. B. G. \& Eysenck, H. J. (1978). Sensation seeking in England and America: cross-cultural, age and sex comparisons. Journal of Consulting Clinical Psychology, 46(1), 139149.

Recebido em janeiro de 2010 Reformulado em abril de 2010 Aprovado em maio de 2010

Zuckerman, M., Buchsbaum, M. S. \& Murphy, D. L. (1980). Sensation seeking and its biological correlates. Psychological Bulletin, 88(1), 187-214.

Sobre os autores:

Valdiney V. Gouveia é professor associado da Universidade Federal da Paraíba e bolsista de produtividade 1B do CNPq. Tem atuado em Psicologia Social e Psicometria, desenvolvendo trabalhos sobre valores, traços de personalidade, comportamentos anti e pró-sociais, e adaptação e construção de instrumentos psicológicos.

Carlos Eduardo Pimentel é mestre em Psicologia (Psicologia Social) pela Universidade Federal da Paraíba (UFPB, 2004) e bolsista de Doutorado do CNPq no Programa de Pós-Graduação em Psicologia Social, do Trabalho e das Organizações (PSTO) da Universidade de Brasilia (UnB). Tem experiência na área de Psicologia, com ênfase em Processos Grupais e de Comunicação, atuando principalmente nos seguintes temas: preferência musical, atitudes, validade de medidas psicológicas e comportamento antissociais.

Rildésia S. V. Gouveia é doutora em Psicologia Social, professora titular do Centro Universitário de João Pessoa, lecionando Psicologia Jurídica. Suas áreas de atuação têm sido problemas escolares, comportamentos antissociais e delitivos, transtornos clínicos infantis e avaliação e medida psicológica.

Leogildo Alves Freires é graduando do curso de psicologia na Universidade Federal da Paraíba e bolsista PIBIC (CNPq / UFPB).

Rebecca Alves Aguiar Athayde é graduanda do curso de Psicologia na Universidade Federal da Paraíba e bolsista PIBIC (CNPq / UFPB).

Rafaella de Carvalho Rodrigues Araújo é graduanda do curso de Psicologia na Universidade Federal da Paraíba e bolsista PROBEX (UFPB).

Este artigo contou com apoio do CNPq por meio de bolsas de Produtividade em Pesquisa e Doutorado concedidas ao primeiro e segundo autores, respectivamente. Os autores agradecem a essa instituição, expressando ainda sua gratidão ao Professor Jeffrey Jensen Arnett por ter disponibilizado o instrumento e permitido sua adaptação. Agradecem também a Gisele Pacheco por ter coletado parte dos dados. 